\title{
Modality and phonological similarity effects in serial recall: Does one's own voice play a role?
}

\author{
SUSAN E. GATHERCOLE and JOHN M. GARDINER \\ City University, London ECIVOHB, England
}

and

\author{
VERNON H. GREGG \\ Birkbeck College, University of London, London WCIE 7HX, England
}

\begin{abstract}
Results of two experiments showed that the modality effect in serial recall of word lists is sharply reduced by high interitem phonological similarity and that the extent of this reduction is much the same irrespective of whether the lists are spoken by the subject or the experimenter. These findings contradict an account of the modality effect recently proposed by Richardson (1979), but the data are entirely consistent with the belief that the effect originates in echoic memory.
\end{abstract}

The normally good recall of the last few items in a list (the recency effect) is improved still further with auditory as opposed to visual presentation. The auditory advantage, which is conventionally referred to as the modality effect, occurs in several paradigms, but it has perhaps been investigated most intensively in serial recall. Many properties of the modality effect, especially in serial recall, are readily explained if it is assumed that in the auditory, unlike the visual, case, sensory information persists long enough to supplement recall (e.g., Crowder \& Morton, 1969; Morton, Crowder, \& Prussin, 1971). One important property of the modality effect in serial recall, and one which is entirely consistent with an acoustic or echoic memory interpretation, is that it is highly vulnerable to phonological similarity among list items. At least four studies have shown that the effect is sharply reduced or eliminated when items within a list are all phonologically similar (Crowder, 1971; Darwin \& Baddeley, 1974; Richardson, 1979; Watkins, Watkins, \& Crowder, 1974). One exceptional finding, however, was also reported by Richardson (1979). He found that, although the modality effect was eliminated by phonological similarity with letters as the list items, with words the variable had no effect. On the basis of these and his other findings, Richardson proposed that, counter to the assumptions of theories of echoic memory, the modality effect has a different ori-

This research was supported by a grant from the Social Science Research Council to the second and third authors and by a linked studentship to the first author. We are particularly grateful to J. T. E. Richardson and D. A. Routh for their helpful criticisms and suggestions. Requests for reprints and for copies of the list materials should be sent to Susan E. Gathercole, who is currently visiting the Department of Psychology, Birkbeck College, University of London, Malet Street, London WC1E 7HX, England. gin in serial recall with words than with letters or other nonlexical items such as digits or syllables. Specifically, the proposal was that the modality effect with words arises from a modality-independent postcategorical lexical storage (PLS) system, and that a system similar to that of the Crowder and Morton (1969) precategorical acoustic store (PAS) gives rise to the modality effect with less meaningful material.

Though consistent with the Crowder (1971) and Darwin and Baddeley (1974) findings of a disruptive effect of similarity on the modality effect in serial recall (the materials they used were syllables), Richardson's (1979) data and theory run counter to the findings reported by Watkins et al. (1974), for, like Richardson, those researchers had used word lists. To account for the discrepancy between his results and theirs, Richardson suggested that differences in the way in which words were presented under auditory conditions might be critical. In Richardson's experiment, the words were read aloud by the experimenter; Watkins et al. had their subjects vocalize visually presented words. Richardson suggested that phonologically similar lists might be particularly difficult for the subjects to vocalize and, moreover, that this difficulty might be cumulative within a list. Thus the modality effect would indeed be sharply reduced by phonological similarity, but only because of the disruption to encoding. We note, albeit in passing, that this notion may also provide an account of Crowder's (1978) finding that the modality effect disappears when subjects vocalize lists of phonologically identical words.

This paper provides further evidence on the effects of phonological similarity on serial recall of auditorily and visually presented word lists, evidence that distinguishes between the two positions which we have now summarized. The first experiment was a straightforward 
test of the generality of earlier findings. Serial recall of phonologically distinct or similar lists of words was compared under conditions in which, using a large, newly constructed set of words, subjects either saw the words or heard them vocalized by the experimenter. If, as suggested by Richardson (1979), the Watkins et al. (1974) result of a reduction in the modality effect with phonologically similar words is due solely to a disruptive effect of subject vocalization, the modality effect here should not be influenced by phonological similarity. Moreover, such an outcome would replicate Richardson's (1979) own result and so lend further support to his model. On the other hand, if the modality effect here is significantly reduced by phonological similarity, the generality of the Watkins et al. (1974) finding would be extended from subject vocalization to experimenter vocalization, and this outcome would fit well with the view that the modality effect with words, as with other types of items, is echoic in origin.

\section{EXPERIMENT 1}

\section{Method}

Subjects. The subjects were 16 students at London University, all of whom spoke English as their first language. They were tested either individually or in pairs and were paid for their services.

Materials. A pool of 256 one-syllable words was constructed such that there were 32 different lists, each containing eight words with a high degree of interitem phonological similarity. Although initially inspired by the materials published by Watkins et al. (1974), for the most part our list items were freely generated by the experimenter with a view simply to having a large and essentially novel set of materials that took account of national peculiarities in usage and pronunciation. The distinctive features among words in the similar lists were the consonant or consonants prior to the vowels; the vowel sounds and the following consonants (where they occurred) were nearly always the same. Examples taken from five representative lists are (1) TAP, GAP, BAP; (2) SLOG, BOG, HOG; (3) WART, TAUGHT, SHORT; (4) GLOW, TOE, WOE; (5) WALL, DRAWL, MAUL. Typical pronunciation of the lists in England is such that they more or less constitute rhyming sets; although a few of the words chosen do not even appear in published word frequency norms, all were familiar to our population of subjects. ${ }^{1}$

For this experiment only 21 similar lists were required, and these were selected arbitrarily from the pool. Twenty-one phonologically distinct lists were obtained by recombining the words from the selected similar lists such that no more than two words in each distinct list were represented in a single similar list. Thus each word appeared in one similar and in one distinct list.

Design. Each subject was tested in all four conditions obtained by combining the two modalities of presentation, auditory or visual, with the two list types, distinct or similar. The lists were presented in four blocks of 10 trials. All lists in each block of trials were presented either auditorily or visually; presentation modality was alternated over blocks, with half of the subjects receiving the first block presented visually and half receiving it auditorily. Within each block there were five similar and five distinct lists. These were randomly ordered with the constraint that no more than two consecutive lists were of the same type. The order of the similar and distinct lists within each block was balanced over two groups of subjects, and the order of the words within each list was reversed for half of the subjects.
Procedure. The experimenter either read aloud each word in the list or showed the subject the word printed on a card, at the rate of 1 word/sec. Following the final word in each list, a visual cue was given that signaled that the subject should commence written recall of the list on the response sheet provided. Subjects were given strict serial recall instructions and were told to guess rather than leave blanks. This instruction was given, perhaps somewhat superstitiously, in order to try to minimize possible response criterion differences. ${ }^{2}$ One practice list was given before each of the first two blocks of trials.

\section{Results}

In the data we report, recall was scored according to a strict, serial, item-in-position criterion. ${ }^{3}$ For statistical purposes we followed a well-established precedent in defining the modality effect in serial recall as the recall advantage to auditory over visual presentation at the last serial position only (e.g., Crowder, 1971; Richardson, 1979; Watkins et al., 1974). Hence, separate statistical analyses are reported for data from the terminal position and from the preterminal positions. It may be noted that our adoption of this particular convention does not materially affect the essential conclusions to be drawn.

The principal results are summarized in Figure 1. As can be seen, there is a large modality effect with distinct lists $[\mathrm{t}(15)=7.66, \mathrm{p}<.001]$ which, although still present $[t(15)=3.87, p<.001]$, appears to be reduced with similar lists. Comparison of individual auditory-visual differences at the terminal serial position for distinct and similar lists showed that this reduction in the modality effect was highly significant $[\mathrm{t}(15)=4.56, \mathrm{p}<.001]$.

Over preterminal positions there was no effect of modality $[F(1,15)<1]$, but both serial position $[F(6,90)=81.15, p<.001]$ and similarity $[F(1,15)=$ $8.92, \mathrm{p}<.01]$ effects were significant, the latter reflecting better recall of distinct than of similar lists. There were no significant interactions between either similarity and serial position $[F(6,90=1.96, p<.05]$ or similarity and modality $[F(1,15)<1]$. The interaction between modality and serial position was significant, however $[F(6,90)=10.90, p<.001]$, which appears to reflect a recall advantage to visual lists over initial

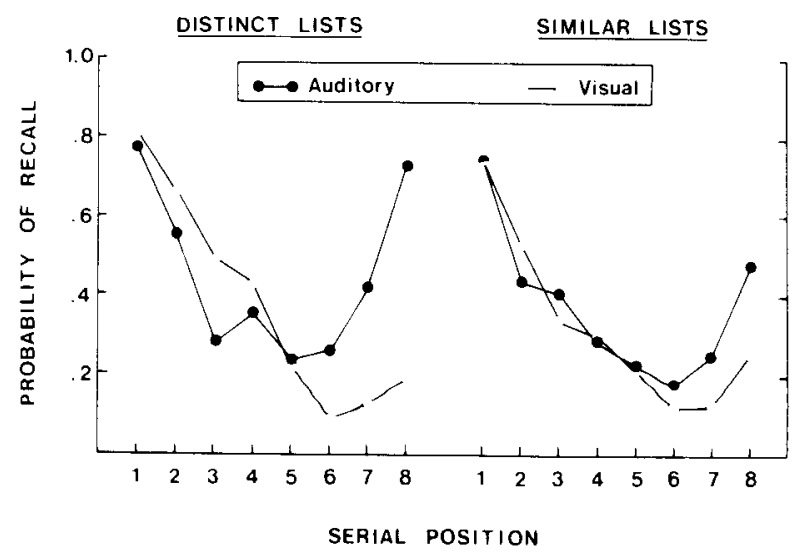

Figure 1. Mean serial recall probabilities: Experiment 1. 
positions and to auditory lists over later positions; the three-way interaction between modality, similarity, and serial position was also significant $[F(6,90)=4.02$, $\mathrm{p}<.005]$.

\section{Discussion}

The modality effect was markedly reduced by phonological similarity. Because this outcome occurred using experimenter vocalization and word lists, it provides no support for Richardson's (1979) suggestion that the similar reduction obtained by Watkins et al. (1974) was due to their use of subject vocalization procedures. The experiment thus extends the generality of the Watkins et al. result and, by the same token, fails to replicate Richardson's result, for he had found no influence of phonological similarity with word lists.

The next experiment was designed to determine whether vocalization by the subject has a disruptive effect on the recall of phonologically similar word lists relative to vocalization by the experimenter. If this were to be the case, it would provide at least some support for Richardson's (1979) suggestion. Hence, Experiment 2 provides a direct comparison of the effects of vocalization by subject and experimenter on the modality effect with lists of phonologically distinct and similar words.

\section{EXPERIMENT 2}

\section{Method}

Subjects. The subjects were 24 London University students, all of whom were tested individually and paid for their services.

Design and Materials. Each subject was tested in all six conditions obtained by combining the three methods of list presentation-experimenter vocalization ("auditory"), subject vocalization ("vocalized"), or visual-with the two list types, distinct and similar. The full pool of 32 different phonologically similar lists was used in this experiment, and 32 phonologically distinct lists were constructed by recombining the words as in Experiment 1.

There were six blocks of 10 trials. Within each block there were five similar and five distinct lists. These were randomly ordered, with the constraint that no more than two consecutive lists were of the same type, and the order of distinct and similar lists was balanced across two groups of subjects. Within a list, word order was reversed for half the subjects. All the lists within each block of 10 trials were presented in the same presentation mode. Each of the six possible orderings of the three modes was used for four subjects over the first three blocks of trials, and these orders were simply repeated over the second three.

Procedure. A similar procedure to that of Experiment 1 was employed for the visual and auditory conditions. In the vocalized condition, the lists were presented as in the visual condition and the subject was required to read each word aloud. One practice trial was given before each of the first three blocks of trials.

\section{Results}

As in Experiment 1, the recall data described were scored by a strict serial criterion. Separate statistical analyses are reported for the data from terminal and preterminal positions.

The principal results are shown in Figure 2. Compar-

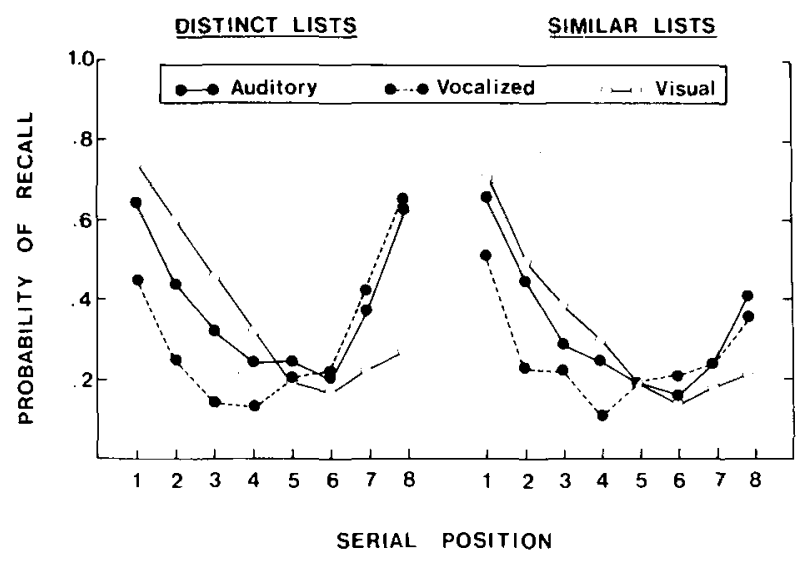

Figure 2. Mean serial recall probabilities: Experiment 2.

ing auditory and visual presentation at the final serial position, there is a large modality effect with distinct lists $[\mathrm{t}(23)=5.86, \mathrm{p}<.001]$ that is still present when similar lists are used $[\mathrm{t}(23)=2.16, \mathrm{p}<.025]$, although considerably reduced. Comparisons of the individual auditory-visual differences at the terminal serial position for the distinct and similar lists showed that this reduction was significant $[t(23)=1.95, p<.05]$. Comparing vocalized and visual presentation, there is also a large modality effect with distinct lists $[\mathrm{t}(23)=7.11$, $p<.001]$, and here, too, the modality effect is still present, although diminished, with similar lists $[t(23)=$ $3.23, \mathrm{p}<.005]$. The reduction in the modality effect with similar lists was significant $[\mathrm{t}(23)=3.22, \mathrm{p}<.005]$. Comparing recall following auditory and vocalized presentation, there was no significant difference in the effects of similarity at the terminal position $[t(23)=$ $1.18, p>.1]$. Nor, within each list type, was there any difference in recall between auditory and vocalized presentation $[\mathrm{t}(23)<1]$ in each case.

Over preterminal positions there were significant main effects of both presentation method $[F(2,46)=$ $17.63, p<.001]$ and serial position $[F(6,138)=27.47$, $p<.001]$. The effect of presentation method appears to be due to the better recall of visual than auditory and of auditory than vocalized lists. The main effect of similarity just missed significance $[F(1,23)=4.02, p=.057]$ : Distinct lists were somewhat better recalled than similar ones. The interaction between presentation method and similarity was not significant $[F(2,46)<1]$. There was a significant interaction between presentation method and serial position $[F(12,276)=10.29, p<.001]$, probably due to the better recall of early list items following visual presentation and of later list items following auditory and vocalized presentation. There was also a significant interaction between similarity and serial position $[F(6,138)=3.03, p<.01]$, which seems to reflect the detrimental effect of similarity being mostly confined to initial and late list positions. The three-way interaction between presentation method, similarity, and serial position was also significant $[F(12,276)=1.98, p<.05]$. 


\section{Discussion}

The results of this experiment replicate and extend those of Experiment 1. The modality effect was again substantially reduced by phonological similarity. Moreover, it was reduced by much the same extent regardless of whether the subject or the experimenter vocalized the words, so there is no evidence that subject vocalization of similar lists leads to a greater reduction in the modality effect than does experimenter vocalization. It is important to note, as well, that the results show that subject vocalization impaired recall performance. But the impairment occurred over preterminal rather than terminal serial positions, ${ }^{4}$ and just as much with distinct as with similar list words.

\section{GENERAL DISCUSSION}

It is widely believed that the modality effect in serial recall originates in echoic memory (e.g., Crowder \& Morton, 1969). One important property of the effect that is entirely consistent with this interpretation is that it is known to be highly vulnerable to phonological similarity among list items (Crowder, 1971; Darwin \& Baddeley, 1974; Richardson, 1979; Watkins et al., 1974; see, too, Crowder, 1978). Richardson (1979), however, found that, although the effect was eliminated when lists of phonologically similar letters were used, with words, similarity had no effect. He proposed that the modality effect with words originates in PLS, a postcategorical lexical storage system, and that with nonlexical material the effect originates in a system similar to Crowder and Morton's PAS (1969). One previous study (Watkins et al., 1974) had, however, found that the modality effect was greatly reduced by high phonological similarity in lists of words. Richardson suggested that the discrepancy between his results and those of Watkins et al. might be due to the latter's use of subject vocalization. Specifically, the notion was that subjects would find it increasingly difficult to vocalize phonologically similar words within a list. By this account, similarity interferes with postcategorical encoding rather than with the storage of echoic information.

Experimental results described here do not provide any support for Richardson's (1979) suggestion. Both experiments showed that the Watkins et al. (1974) result may be readily obtained when the experimenter rather than the subject vocalizes the words. Furthermore, Experiment 2 showed that phonological similarity reduced the modality effect by much the same extent, irrespective of whether the experimenter or the subject vocalized the words. Given these findings, the account of the modality effect in serial recall provided by Richardson's PLS model must be rejected. Instead, we conclude that our results are entirely consistent with previous echoic memory interpretations of the effect (e.g., Crowder \& Morton, 1969).

Why, then, did Richardson's (1979) experiment reveal no influence of phonological similarity on the modality effect with word lists, only with lists of letters? We cannot say for sure. But inspection of his data shows that the level of recency recall was particularly high following auditory presentation of lists of distinct words and letters (Richardson, 1979, Figure 1). And we have some evidence that strongly suggests that performance in the recall of distinct auditory word lists may have been constrained by a ceiling effect. Though we have not collected evidence in the case of distinct auditory letter lists, the same thing may be true there. Be that as it may, our evidence implies that, had the level of recency recall been lower in Richardson's experiment, the magnitude of the modality effect with distinct word lists would then have been great enough to produce a significant reduction in the effect with similar word lists-in accord with both our own and the Watkins et al. (1974) results. The relevant evidence comes from two unpublished experiments (Gathercole, Gregg, \& Gardiner, Note 1). Richardson had used precisely the same set of six words for each word list, and we used his distinct word set to provide a "standard comparison" in each experiment. In one, we compared the extent of the modality effect obtained with those items to that obtained with lists made up from another constant set of 10 phonologically distinct words. In the other, we made the comparison with kists made up from a quite different set of six distinct words on each trial. In both experiments we observed a higher level of recall generally under the conditions used by Richardson, and particularly so in the case of the terminal item in auditory lists. More important, in both experiments the modality effect was significantly greater under the other conditions we introduced than under those used by Richardson.

Finally, although our results are indeed entirely consistent with an echoic interpretation of the modality effect, it should also be acknowledged that such interpretations have been challenged recently by results of a number of other studies, including some from our own laboratories. At the moment it is perhaps not altogether clear how any current controversy over echoic memory interpretations of the modality effect will eventually be resolved, and the present study does not provide evidence which bears directly on that issue. It does, however, demonstrate that, at least with respect to modality and phonological similarity effects in serial recall of word lists, the subject's own voice seems functionally equivalent to the experimenter's voice. Hence, contrary to Richardson's (1979) PLS model, there now seems little basis for supposing that the lexical nature of the to-be-remembered items is of any importance to understanding the modality effect.

\section{REFERENCE NOTE}

1. Gathercole, S. E., Gregg, V. H., Gardiner, J. M. The modality effect and echoic memory. Paper presented at the London Conference of the British Psychological Society, December 1980. 


\section{REFERENCES}

Crowder, R. G. The role of one's own voice in immediate memory. Cognitive Psychology, 1970, 1, 157-178.

Crowder, R. G. The sounds of vowels and consonants in immediate memory. Journal of Verbal Learning and Verbal Behavior, $1971,10,587-596$.

Crowder, R. G. Memory for phonologically uniform lists. Journal of Verbal Learning and Verbal Behavior, 1978, 17, 73-89.

Crowder, R. G. Similarity and order in memory. In G. H. Bower (Ed.), The psychology of learning and motivation: Advances in research and theory (Vol. 13). New York: Academic Press, 1979.

Crowder, R. G., \& Morton, J. Precategorical acoustic storage (PAS). Perception \& Psychophysics, 1969, 5, 365-373.

Darwin, C. J., \& Baddeley, A. D. Acoustic memory and the perception of speech. Cognitive Psychology, 1974, 6, 41-60.

Morton, J., Crowder, R. G., \& Prussin, H. A. Experiments with the stimulus suffix effect. Journal of Experimental Psychology, 1971, 91, 169-190.

Richardson, J. T. E. Precategorical acoustic storage and postcategorical lexical storage. Cognitive Psychology, 1979, 11, 265-286.

Routh, D. A. "Trace strength," modality, and the serial position curve in immediate memory. Psychonomic Science, 1970, 18, 355-357.

Watkins, M. J., Watkins, O. C., \& Chowden, R. G. The modality effect in free and serial recall as a function of phonological similarity. Journal of Verbal Learning and Verbal Behavior, 1974, 13, 430-447.

\section{NOTES}

1. Copies of the lists are available on request, although, for the reasons explained, they may be of limited use to any but researchers in England.

2. The percentage of omissions was essentially the same for distinct and similar lists both in Experiment 1 (30\% and 27\%, respectively) and in Experiment $2(36 \%$ and $34 \%$, respectively).

3 . The results of this and of the following experiment were also scored according to a free recall criterion. We are grateful to an anonymous referee who drew our attention to the considerable difficulties entailed in interpreting those data. However, we note that there was a marked facilitatory effect of high intralist similarity in our free-scored data and also that similarity reduced the modality effect there too, a pattern of results not unlike that obtained by Watkins et al. (1974). For further discussion, particularly of the more general effects of similarity on recall, see Crowder (1979) and Watkins et al.

4. Crowder (1970) reported a comparable decrement over preterminal positions in serial recall of digits and also found that subject and experimenter voices were equivalent with respect to the modality effect. However, there are also cases in which subject vocalization apparently led to little or no poorer recall than visual presentation (e.g., Routh, 1970; Watkins et al., 1974). When an impairment is found, it seems quite likely that it is indeed due to a disruption of normal encoding processes.

(Received for publication June 12, 1981; revision accepted November 11,1981 .) 